\title{
A 10- or 14-day Bismuth-containing Quadruple Therapy as a First-line Helicobacter pylori Eradication Therapy: A Systematic Review and Meta-analysis
}

\begin{abstract}
Sung Eun Kim ${ }^{1}$, Hye-Kyung Jung ${ }^{2}$, Seung Joo Kang ${ }^{3}$, Yong Chan Lee ${ }^{4}$, Hyo-Joon Yang ${ }^{5}$, Seon-Young Park ${ }^{6}$, Cheol Min Shin ${ }^{7}$, Hyun Chul Lim ${ }^{8}$, Jie-Hyun Kim ${ }^{9}$, Su Youn Nam ${ }^{10,11}$, Woon Geon Shin ${ }^{12}$, Jae Myung Park ${ }^{13}$, Il Ju Choi ${ }^{14}$, Jae Gyu Kim ${ }^{15}$, Miyoung Choi ${ }^{16}$; Korean College of Helicobacterand Upper Gastrointesinal Research

Department of Internal Medicine, Kosin University College of Medicine ${ }^{1}$, Busan, Department of Internal Medicine, Ewha Womans University School of Medicine ${ }^{2}$, Department of Internal Medicine, Seoul National University Hospital Gangnam Center ${ }^{3}$, Department of Internal Medicine, Yonsei University College of Medicine ${ }^{4}$, Division of Gastroenterology, Department of Internal Medicine and Gastrointestinal Cancer Center, Kangbuk Samsung Hospital, Sungkyunkwan University School of Medicine ${ }^{5}$, Seoul, Department of Internal Medicine, Chonnam National University Medical School ${ }^{6}$, Gwangju, Department of Internal Medicine, Seoul National University Bundang Hospital $7^{7}$, Seongnam, Department of Internal Medicine, Yongin Severance Hospital, Yonsei University College of Medicine ${ }^{8}$, Yongin, Department of Internal Medicine and Institute of Gastroenterology, Yonsei University College of Medicine ${ }^{9}$, Seoul, Department of Gastroenterology, Center for Gastric Cancer, Kyungpook National University Hospital Chilgok Hospital ${ }^{80}$, Department of Gastroenterology, School of Medicine, Kyungpook National University ${ }^{11}$, Daegu, Department of Internal Medicine, Hallym University College of Medicine ${ }^{12}$, Division of Gastroenterology and Hepatology, Department of Internal Medicine, Seoul St. Mary's Hospital, The Catholic University of Korea ${ }^{13}$, Seoul, Center for Gastric Cancer, National Cancer Center ${ }^{14}$, Goyang, Department of Internal Medicine, Chung-Ang University College of Medicine ${ }^{15}$, Division of Healthcare Technology Assessment Research, National Evidence-based Healthcare Collaborating Agency ${ }^{16}$, Seoul, Korea
\end{abstract}

Background/Aims: The eradication rate of the first-line standard triple therapy (STT) for Helicobacter pylori (H. pylori) infection has decreased since 2000; therefore, other first-line therapies are required. This study was aimed at investigating the efficacy of bismuth-containing quadruple therapy (PBMT) for first-line H. pylori eradication compared to STT, sequential therapy (SQT), and concomitant therapy (CT).

Materials and Methods: The Ovid-MEDLINE, Koreamed, EMBASE, KMBASE, and Cochrane Library databases were searched from January 2008 to July 2018. All identified randomized controlled trials (RCTs) comparing PBMT and non-PBMT for first-line H. pylori eradication therapy were included in the final analysis.

Results: A total of 3,653 patients from seven RCTs were enrolled. The pooled eradication rates of PBMT by intention-to-treat (ITT) and per-protocol (PP) analyses were 82.1\% (95\% CI, 68.2 90.8\%) and 88.8\% (95\% CI, 77.1 94.9\%), respectively. However, no statistically significant difference was observed in eradication rates of the 10- or 14-day PBMT as compared to 14-day STT, 10-day SQT, and 10-day CT in ITT and PP analyses. PBMT was significantly higher in adverse events than in the other eradication regimens $(\mathrm{RR}, 1.64 ; 95 \% \mathrm{CI}, 1.11 \sim 2.44)$. Considerable heterogeneity in adverse events was observed among studies $\left(\chi^{2}=88.7 ; P<0.001\right.$, $\left.I^{2}=93 \%\right)$.

Conclusions: PBMT can be the first-line treatment for $H$. pylori eradication in Korea when other first-line options, including STT, SQT, or CT, are unavailable due to their high adverse event rates. (Korean J Helicobacter Up Gastrointest Res 2021;21:48-58)

Key Words: Adverse effects; Bismuth tripotassium dicitrate; Disease eradication; Helicobacter pylori; Meta-analysis

\section{INTRODUCTION}

As of 2015, the global prevalence of Helicobacter pylori (H. pylori) was approximately 4.4 billion. ${ }^{1}$ This common infection is well known to cause gastritis, peptic ulcers, gastric mucosa-associated lymphoid tissue lympho-

Received: October 13, 2020 Revised: November 6, 2020 Accepted: November 10, 2020 Corresponding author: Sung Eun Kim

Department of Internal Medicine, Kosin University College of Medicine, 262 Gamcheon-ro, Seo-gu, Busan 49267, Korea

Gamcheon-ro, Seo-gu, Busan 49267, Korea
Tel: 82-51-990-5225, Fax: +82-51-990-5055, E-mail: solefide@ $@$ hanmail.net ma, and gastric cancer. ${ }^{2}$ Therefore, effective eradication therapy is crucial for building public health, particularly in high $H$. pylori prevalence areas, including Korea.

According to Korean guidelines for the diagnosis and treatment of $H$. pylori infection, which were revised in 2013, standard triple therapy, comprising proton pump inhibitors (PPIs), amoxicillin, and clarithromycin, is the recommended first-line eradication regimen for $H$. pylori infection. ${ }^{3}$ Unfortunately, the efficacy of the standard triple therapy is declining worldwide, and this tendency is also observed in Korea. ${ }^{4-6}$ The H. pylori eradication rates

Copyright $\odot 2021$ Korean College of Helicobacter and Upper Gastrointestinal Research

(a) The Korean Journal of Helicobacter and Upper Gastrointestinal Research is an Open-Access Journal. All articles are distributed under the terms of the Creative Commons Attribution Non-Commercial License (http:// creativecommons.org/licenses/by-nc/4.0) which permits unrestricted non-commercial use, distribution, and reproduction in any medium, provided the original work is properly cited. 
in Korea using standard triple therapy were 84.9 87.5\% from 2001 to 2007 and $80.0 \sim 81.4 \%$ from 2008 to 2010 $(P<0.0001)$, presenting a decreasing trend for the last 10 years. ${ }^{5}$

Several recognized guidelines recommended that standard triple therapy, sequential therapy (SQT), concomitant therapy (CT), bismuth-containing quadruple, or non-bismuth-containing quadruple therapies are a recommended first-line treatment option. ${ }^{7,8}$ In the aforementioned revised Korean guidelines, when clarithromycin resistance is suspected, bismuth-containing quadruple therapy is used as the first-line therapy; however, it is generally recommended as a second-line regimen to eradicate $H$. pylori. ${ }^{3}$

Bismuth-containing quadruple therapy is an effective regimen for $H$. pylori eradication. However, the efficacy of bismuth-containing quadruple therapy as a first-line treatment has depended on the type of eradication regimens and duration of therapies in two network meta-analyses of the randomized controlled trials (RCTs). ${ }^{9,10}$ With this background, a meta-analysis was conducted to evaluate the efficacy and safety of bismuth-containing quadruple therapy for first-line $H$. pylori eradication and compare it to standard triple therapy, SQT, and CT, which are commonly used as first-line eradication therapies.

\section{MATERIALS AND METHODS}

\section{Search strategy}

We searched Ovid-MEDLINE, KoreaMed, EMBASE, KMBASE, and Cochrane Library from January 2008 to July 2018. The exploring key words were "Helicobacter pylori or Helicobacter infection" and "bismuth based quadruple therapy, bismuth therapy, or bismuth treat". Languages were limited to English and Korean. The reporting of the current study follows the PRISMA guidelines. ${ }^{11}$

\section{Study selection}

Studies were included in the meta-analysis if the following criteria were met: 1) RCTs; 2) bismuth-containing quadruple therapy as a first-line eradication therapy; 3) $H$. pylori infection was identified by at least one of the fol- lowing modalities: rapid urease test, urea breath test, histology, or culture; 4) successful eradication of $H$. pylori infection was clarified with previous mentioned modalities; and 5) bismuth-containing quadruple therapy consisting of PPIs, bismuth compounds, metronidazole, and tetracycline.

Non-RCTs, studies with second-line treatment using bismuth-containing quadruple therapy, bismuth-containing regimens including Pylera ${ }^{\mathbb{R}}$ (Allegran, Dublin, Ireland), control groups consisting of the same regimens as the intervention group with different duration or different doses, reviews, case reports, letters, editorials, and abstracts were excluded.

Two investigators (S.E.K. and H.K.J.) separately reviewed the results of the search, and selected the included studies. Disagreement was resolved by consensus.

\section{Data extraction}

The following information was collected from the enrolled studies: 1) name of the first author, year of publication, country, and trial types; 2) number of subjects in each study according to therapeutic regimens, mean age, therapeutic regimens, duration of regimens, and funding; 3) diagnostic methods of confirming $H$. pylori infection and eradication, and eradication follow-up period; and 4) number of subjects who were successfully treated in each regimen, eradication rates of both intention-to-treat (ITT) and per-protocol (PP) analyses, and adverse events.

\section{Risk of bias}

Two investigators (S.E.K. and H.K.J.) independently assessed the potential bias of enrolled studies using Cochrane Handbook for Systemic Reviews in Intervention. ${ }^{12}$ The assessment tool of Cochrane Collaboration was categorized as randomization, allocation concealment, blinding of participants and personnel, blinding of outcome assessment, incomplete outcome data, and selective outcome data.

\section{Statistical analysis}

All statistical analyses were performed with Review 
Manager version 5.3 software (Revman; The Cochrane Collaboration Oxford, UK). The pooled eradication rate with 95\% CI in bismuth-containing quadruple therapy and non-bismuth-containing quadruple therapy was calculated. Thereafter, subgroup analyses were conducted according to the eradication regimens. The assessments of the subgroup analyses and adverse events were expressed as the RR with 95\% CI. We investigated heterogeneity between studies by using $I^{2}$ statistics. We considered $I^{2}$ value of $25 \%, 50 \%$, and $75 \%$ to be low, moderate, and high degrees of heterogeneity, respectively. When high heterogeneity occurred, we calculated RRs using random-effect models. We used both ITT and PP analyses for clinical outcomes. A $P<0.05$ was suggested as being statistically significant.

\section{RESULTS}

\section{Study selection}

A total of 204 studies were reviewed. Fig. 1 shows the flow diagram of the literature search. Subsequently, we excluded 172 studies after reviewing the title or abstract, and we reviewed full articles or abstracts for 32 studies. Among them, 25 studies were excluded according to the selection criteria. Finally, seven RCTs with 3,653 patients were enrolled for meta-analysis. ${ }^{13-19}$ The study design of five trials was multiple arms; therefore, the data of the groups that accorded with the inclusion criteria were selected and included for analysis.

\section{Characteristics of studies and risk of bias}

Table 1 presents the main characteristics of the studies. All studies used a first-line bismuth-containing quadruple therapy consisting of PPIs (pantoprazole, rabeprazole, or lansoprazole), bismuth compounds (bismuth subcitrate, bismuth tripotassium dicitrate, bismuth potassium citrate, colloidal bismuth subcitrate, or bismuth subsalicylate), metronidazole, and tetracycline (PBMT). Patients who received PBMT were 1,206, and 2,447 patients were treated with non-PBMT regimens. Among the non-PBMT regimen groups, 753 patients of three trials in standard triple therapy group, 230 patients in two trials in the SQT group, and 770 patients of three trials in the CT group were eli-

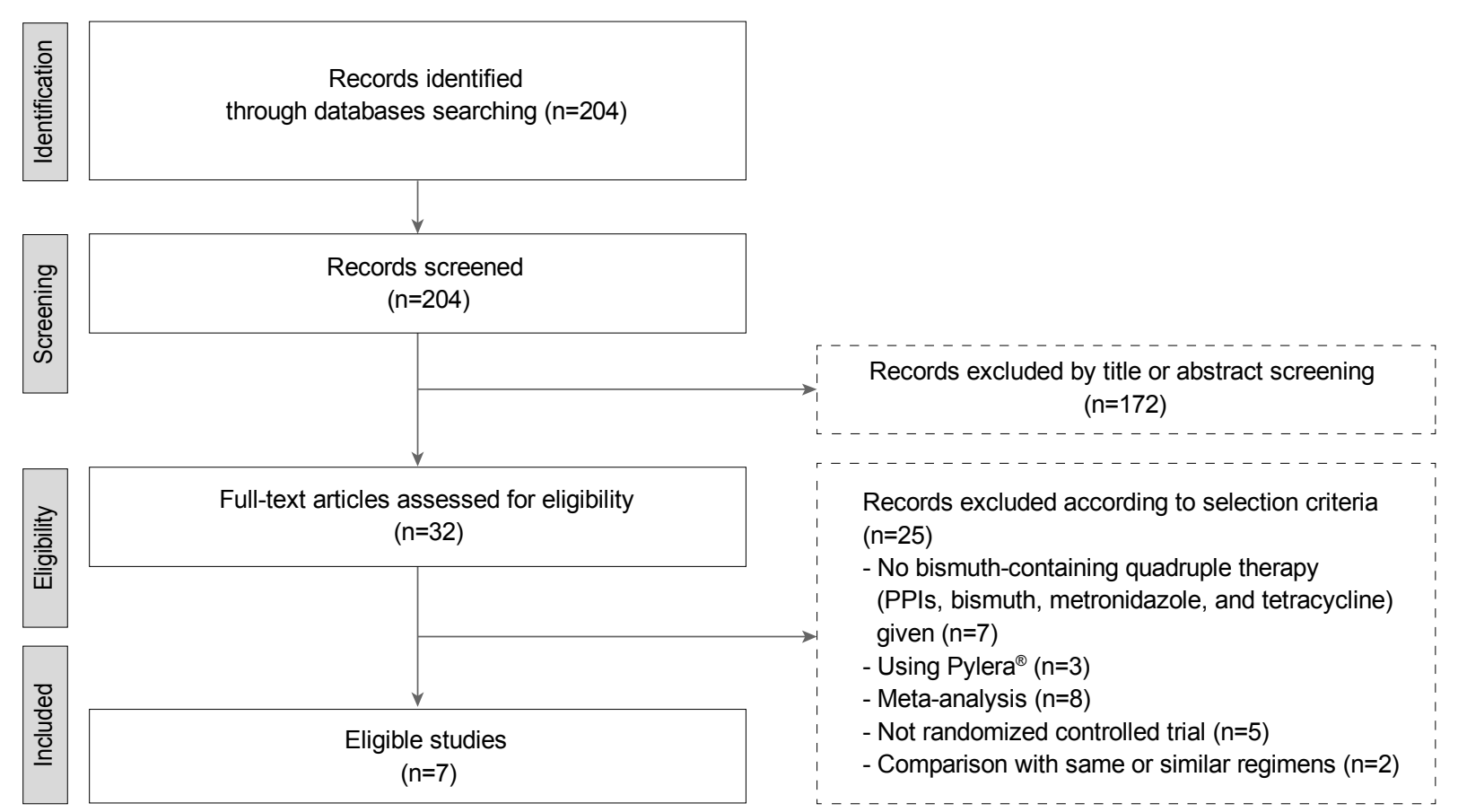

Fig. 1. Preferred Reporting Items for Systematic Reviews and Meta-Analysis (PRISMA) flow diagram of the study selection. PPIs, proton pump inhibitors. 
Table 1. Characteristics of the Studies Included in the Meta-analysis

\begin{tabular}{|c|c|c|c|c|c|c|c|}
\hline Study & Nation & $\begin{array}{l}\text { Bismuth-based } \\
\text { regimens }\end{array}$ & $\begin{array}{l}\text { Bismuth-based } \\
\text { regimens } \\
\text { duration } \\
\text { (days) }\end{array}$ & Control regimens & $\begin{array}{c}\text { Control } \\
\text { regimens } \\
\text { duration } \\
\text { (days) }\end{array}$ & $\begin{array}{c}\text { Test to } \\
\text { diagnose } \\
\text { Helicobacter } \\
\text { pylori }\end{array}$ & $\begin{array}{c}\text { Test to } \\
\text { confirm } \\
\text { eradication }\end{array}$ \\
\hline $\begin{array}{c}\text { Tsay et al. }^{13} \\
\text { (2017) }\end{array}$ & Taiwan & $\begin{array}{l}\text { Pantoprazole } 40 \mathrm{mg} \\
\text { bid, bismuth } \\
\text { subcitrate } 120 \mathrm{mg} \\
\text { qid, metronidazole } \\
250 \mathrm{mg} \text { qid, } \\
\text { tetracycline } 500 \mathrm{mg} \\
\text { qid (n=164) }\end{array}$ & 14 & $\begin{array}{l}\text {-First } 7 \text { days: pantoprazole } 40 \mathrm{mg} \text { bid, } \\
\text { amoxicillin } 1 \mathrm{~g} \text { bid } \\
\text {-Second } 7 \text { days: pantoprazole } 40 \mathrm{mg} \text { bid, } \\
\text { amoxicillin } 1 \mathrm{~g} \text { bid, clarithromycin } 500 \mathrm{mg} \\
\text { bid, metronidazole } 500 \mathrm{mg} \text { bid }(\mathrm{n}=166)\end{array}$ & 14 & $\begin{array}{l}\text { RUT, } \\
\text { histology, } \\
\text { culture }\end{array}$ & $\begin{array}{l}\text { RUT, } \\
\text { histology }\end{array}$ \\
\hline $\begin{array}{l}\text { Sapmaz et } \\
\text { al. }^{14} \\
(2017)\end{array}$ & Turkey & $\begin{array}{l}\text { Rabeprazole } 20 \mathrm{mg} \text { bid, } \\
\text { bismuth subcitrate } \\
120 \text { mg qid, } \\
\text { metronidazole } 500 \\
\text { mg tid, tetracycline } \\
500 \mathrm{mg} \text { qid }(\mathrm{n}=100)\end{array}$ & 14 & $\begin{array}{l}\text { Rabeprazole } 20 \mathrm{mg} \text { tid, amoxicillin } 750 \mathrm{mg} \\
\text { tid }(\mathrm{n}=100)\end{array}$ & 14 & Histology & $\begin{array}{l}\text { Stool } \\
\text { antigen } \\
\text { test }\end{array}$ \\
\hline $\begin{array}{l}\text { Liou et al. } \\
\text { (2016) }\end{array}$ & Taiwan & $\begin{array}{l}\text { Lansoprazole } 30 \mathrm{mg} \\
\text { bid, bismuth } \\
\text { tripotassium } \\
\text { dicitrate } 300 \mathrm{mg} \text { qid, } \\
\text { metronidazole } 500 \\
\text { mg tid, tetracycline } \\
500 \mathrm{mg} \text { qid }(\mathrm{n}=540)\end{array}$ & 10 & $\begin{array}{l}\text { Group 1: lansoprazole } 30 \mathrm{mg} \text { bid, } \\
\text { amoxicillin } 1 \text { g bid, clarithromycin } 500 \mathrm{mg} \\
\text { bid, metronidazole } 500 \mathrm{mg} \text { bid }(\mathrm{n}=540) \\
\text { Group 2: lanroprazole } 30 \mathrm{mg} \text { bid, } \\
\text { amoxicillin } 1 \mathrm{~g} \text { bid, clarithromycin } 500 \mathrm{mg} \\
\text { bid }(\mathrm{n}=540)\end{array}$ & $\begin{array}{l}\text { Group 1: } 10 \\
\text { Group 2: } 14\end{array}$ & $\begin{array}{l}\text { UBT, RUT, } \\
\text { histology, } \\
\text { culture, } \\
\text { serology }\end{array}$ & UBT \\
\hline $\begin{array}{l}\text { Kefeli et } \\
\text { al. }^{16} \\
(2016)\end{array}$ & Turkey & $\begin{array}{l}\text { Rabeprazole } 40 \mathrm{mg} \text { bid, } \\
\text { bismuth potassium } \\
\text { citrate } 220 \mathrm{mg} \text { bid, } \\
\text { metronidazole } 500 \\
\text { mg tid, tetracycline } \\
500 \mathrm{mg} \text { tid }(\mathrm{n}=130)\end{array}$ & 10 & $\begin{array}{l}\text { Group } 1(\mathrm{n}=130) \text { : } \\
\text {-First } 5 \text { days: rabeprazole } 40 \mathrm{mg} \text { bid, } \\
\text { amoxicillin } 1 \mathrm{~g} \text { bid } \\
\text {-Second } 5 \text { days: rabeprazole } 40 \mathrm{mg} \text { bid, } \\
\text { clarithromycin } 500 \mathrm{mg} \text { bid, metronidazole } \\
500 \mathrm{mg} \text { tid } \\
\text { Group } 2(\mathrm{n}=130) \text { : rabeprazole } 40 \mathrm{mg} \text { bid, } \\
\text { amoxicillin } 1 \mathrm{~g} \text { bid, clarithromycin } 500 \mathrm{mg} \\
\text { bid, metronidazole } 500 \mathrm{mg} \text { tid }\end{array}$ & $\begin{array}{l}\text { Group 1: } 10 \\
\text { Group 2: } 10\end{array}$ & Histology & UBT \\
\hline $\begin{array}{l}\text { Gokcan et } \\
\text { al. }^{17} \\
(2016)\end{array}$ & Turkey & $\begin{array}{l}\text { Lansoprazole } 30 \mathrm{mg} \\
\text { bid, colloidal } \\
\text { bismuth subcitrate } \\
300 \text { mg qid, } \\
\text { metronidazole } 500 \\
\text { mg tid, tetracycline } \\
500 \text { mg qid }(n=53)\end{array}$ & 14 & $\begin{array}{l}\text { Group } 1(\mathrm{n}=42) \text { : lanroprazole } 30 \mathrm{mg} \text { bid, } \\
\text { colloidal bismuth subcitrate } 300 \mathrm{mg} \text { qid, } \\
\text { amoxicillin } 1 \mathrm{~g} \text { bid, levofloxacin } 500 \mathrm{mg} \text { qd } \\
\text { Group } 2(\mathrm{n}=54) \text { : lanroprazole } 30 \mathrm{mg} \text { bid, } \\
\text { colloidal bismuth subcitrate } 300 \mathrm{mg} \text { qid, } \\
\text { amoxicillin } 1 \mathrm{~g} \text { bid, clarithromycin } 500 \mathrm{mg} \\
\text { bid }\end{array}$ & $\begin{array}{l}\text { Group 1: } 14 \\
\text { Group 2: } 14\end{array}$ & $\begin{array}{l}\text { UBT, } \\
\text { histology }\end{array}$ & $\begin{array}{l}\text { UBT, } \\
\text { histology }\end{array}$ \\
\hline $\begin{array}{l}\text { Gungor et } \\
\text { al. }^{18} \\
(2015)\end{array}$ & Turkey & $\begin{array}{l}\text { Pantoprazole } 40 \mathrm{mg} \\
\text { bid, bismuth } \\
\text { subsalicylate } 300 \mathrm{mg} \\
\text { qid, metronidazole } \\
500 \mathrm{mg} \text { tid, } \\
\text { tetracycline } 500 \mathrm{mg} \\
\text { qid (n=100) }\end{array}$ & 14 & $\begin{array}{l}\text { Group } 1(\mathrm{n}=100) \text { : pantoprazole } 40 \mathrm{mg} \text { bid, } \\
\text { amoxicillin } 1 \mathrm{~g} \text { bid, clarithromycin } 500 \mathrm{mg} \\
\text { bid } \\
\text { Group } 2(\mathrm{n}=100) \text { : pantoprazole } 40 \mathrm{mg} \text { bid, } \\
\text { amoxicillin } 1 \mathrm{~g} \text { bid, metronidazole } 500 \mathrm{mg} \\
\text { bid } \\
\text { Group } 3 \text { ( } \mathrm{n}=100) \text { : } \\
\text {-First } 5 \text { days: pantoprazole } 40 \mathrm{mg} \text { bid, } \\
\text { amoxicillin } 1 \mathrm{~g} \text { bid } \\
\text {-Second } 5 \text { days: pantoprazole } 40 \mathrm{mg} \text { bid, } \\
\text { tetracycline } 500 \text { mg qid, metronidazole } \\
500 \mathrm{mg} \text { bid } \\
\text { Group } 4(\mathrm{n}=100) \text { : pantoprazole } 40 \mathrm{mg} \text { bid, } \\
\text { amoxicillin } 1 \mathrm{~g} \text { bid, tetracycline } 500 \mathrm{mg} \mathrm{qid,} \\
\text { metronidazole } 500 \text { mg bid }\end{array}$ & $\begin{array}{l}\text { Group 1: } 14 \\
\text { Group 2: } 14 \\
\text { Group 3: } 10 \\
\text { Group 4: } 10\end{array}$ & $\begin{array}{l}\text { UBT, stool } \\
\text { antigen } \\
\text { test, RUT, } \\
\text { histology }\end{array}$ & UBT \\
\hline
\end{tabular}


Table 1. Continued

\begin{tabular}{|c|c|c|c|c|c|c|c|}
\hline Study & Nation & $\begin{array}{l}\text { Bismuth-based } \\
\text { regimens }\end{array}$ & $\begin{array}{l}\text { Bismuth-based } \\
\text { regimens } \\
\text { duration } \\
\text { (days) }\end{array}$ & Control regimens & $\begin{array}{l}\text { Control } \\
\text { regimens } \\
\text { duration } \\
\text { (days) }\end{array}$ & $\begin{array}{c}\text { Test to } \\
\text { diagnose } \\
\text { Helicobacter } \\
\text { pylori }\end{array}$ & $\begin{array}{c}\text { Test to } \\
\text { confirm } \\
\text { eradication }\end{array}$ \\
\hline $\begin{array}{l}\text { Songür et } \\
\text { al. }^{19} \\
(2009)\end{array}$ & Turkey & $\begin{array}{l}\text { Lansoprazole } 30 \mathrm{mg} \\
\text { bid, bismuth } \\
\text { subcitrate } 300 \mathrm{mg} \\
\text { qid, metronidazole } \\
500 \mathrm{mg} \text { bid, } \\
\text { tetracycline } 500 \mathrm{mg} \\
\text { qid ( } \mathrm{n}=119)\end{array}$ & 10 & $\begin{array}{l}\text { Group } 1(\mathrm{n}=113) \text { : lanroprazole } 30 \mathrm{mg} \text { bid, } \\
\text { amoxicillin } 1 \mathrm{~g} \text { bid, clarithromycin } 500 \mathrm{mg} \\
\text { bid } \\
\text { Group } 2(\mathrm{n}=117) \text { : lanroprazole } 30 \mathrm{mg} \text { bid, } \\
\text { ranitidine bismuth citrate } 400 \mathrm{mg} \text { bid, } \\
\text { tetracycline } 500 \mathrm{mg} \text { qid, metronidazole } \\
500 \mathrm{mg} \text { bid } \\
\text { Group } 3 \text { ( } \mathrm{n}=115) \text { : lanroprazole } 30 \mathrm{mg} \text { bid, } \\
\text { tetracycline } 500 \mathrm{mg} \text { qid, metronidazole } \\
500 \mathrm{mg} \text { bid }\end{array}$ & $\begin{array}{l}\text { Group 1: } 14 \\
\text { Group 2: } 10 \\
\text { Group 3: } 10\end{array}$ & $\begin{array}{l}\text { UBT, } \\
\text { histology }\end{array}$ & UBT \\
\hline
\end{tabular}

bid, twice a day; qid, four times a day; RUT, rapid urease test; tid, three times a day; UBT, urea breath test; qd, once a day.

\begin{tabular}{|c|c|c|c|c|c|c|c|c|c|}
\hline \multirow[b]{2}{*}{ Study or subgroup } & \multicolumn{2}{|c|}{ 10-/14-day PBMT } & \multicolumn{2}{|c|}{ 14-day STT } & \multirow{2}{*}{$\begin{array}{c}\text { Weight } \\
(\%)\end{array}$} & \multirow{2}{*}{$\begin{array}{c}\text { Risk ratio } \\
\mathrm{M}-\mathrm{H}, \text { random, } 95 \% \mathrm{Cl}\end{array}$} & \multirow{2}{*}{\multicolumn{2}{|c|}{$\begin{array}{c}\text { Risk ratio } \\
\mathrm{M}-\mathrm{H}, \text { random, } 95 \% \mathrm{Cl}\end{array}$}} & \multirow{2}{*}{$\begin{array}{l}\text { Risk of bias } \\
\text { A B C D E F G }\end{array}$} \\
\hline & \multirow{2}{*}{$\begin{array}{c}\text { Events } \\
62\end{array}$} & \multirow{2}{*}{$\begin{array}{c}\text { Total } \\
100\end{array}$} & \multirow{2}{*}{$\frac{\text { Events }}{42}$} & \multirow{2}{*}{$\begin{array}{c}\text { Total } \\
100\end{array}$} & & & & & \\
\hline Gungor et al. $^{18}(2015)$ & & & & & 30.4 & $1.48(1.12,1.95)$ & & \multirow{3}{*}{ - } & \multirow{5}{*}{ 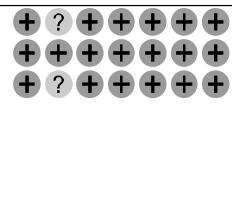 } \\
\hline Liou et al. ${ }^{15}(2016)$ & 488 & 540 & 452 & 540 & 42.3 & $1.08(1.03,1.13)$ & & & \\
\hline Songür et al. ${ }^{19}$ (2009) & 56 & 119 & 37 & 113 & 27.3 & $1.44(1.04,1.99)$ & & & \\
\hline Total $(95 \% \mathrm{Cl})$ & & 759 & & 753 & 100.0 & $1.28(0.97,1.70)$ & & 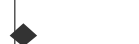 & \\
\hline Total events & 606 & & 531 & & & & & & \\
\hline \multicolumn{7}{|c|}{ Heterogeneity: $\mathrm{Tau}^{2}=0.05, \mathrm{chi}^{2}=10.14, \mathrm{df}=2(P=0.006), I^{2}=80 \%$} & 0.01 & 10 & \\
\hline \multicolumn{7}{|c|}{ Test for overall effect: $Z=1.73(P=0.08)$} & $\begin{array}{r}\text { Favours 10-14-day } \\
\text { PBMT }\end{array}$ & \multicolumn{2}{|c|}{ Favours 14-day STT } \\
\hline
\end{tabular}

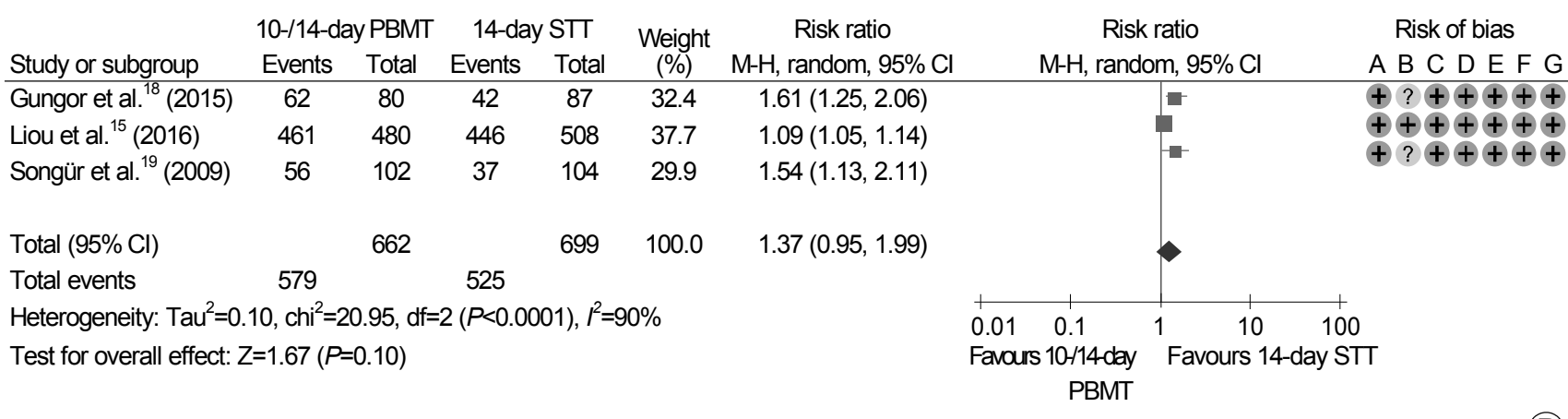

Fig. 2. Forest plot of Helicobacter pylori eradication of 10- or 14-day bismuth-containing quadruple therapy (PMBT) vs. 14-day standard triple therapy (STT) as the first-line therapy. (A) Intention-to-treat analysis. (B) Per-protocol analysis. Risk of bias: A, random sequence generation (selection bias); B, allocation concealment (selection bias); C, blinding of participants and personnel (performance bias); D, blinding of outcome assessment (detection bias); E, incomplete outcome data (attrition bias); F, selective reporting (reporting bias); G, other bias. M-H, Mantel-Haenszel test.

gible to subgroup analysis for eradication efficacy. The risks of bias assessment in seven trials are shown in Supplementary Table 1.

\section{Eradication rates of PBMT}

The pooled eradication rates of PBMT were $82.1 \%$ (95\% CI, 68.2 90.8\%) in ITT analysis and 88.8\% (95\% CI, 


\begin{tabular}{|c|c|c|c|c|c|c|c|c|c|c|}
\hline \multirow[b]{2}{*}{ Study or subgroup } & \multicolumn{2}{|c|}{ 10-/14-day PBMT } & \multicolumn{2}{|c|}{ 10-day SQT } & \multirow{2}{*}{$\begin{array}{c}\text { Weight } \\
(\%)\end{array}$} & \multirow{2}{*}{$\begin{array}{c}\text { Risk ratio } \\
\mathrm{M}-\mathrm{H}, \text { random, } 95 \% \mathrm{Cl}\end{array}$} & \multirow{2}{*}{\multicolumn{3}{|c|}{$\begin{array}{c}\text { Risk ratio } \\
\mathrm{M}-\mathrm{H}, \text { random, } 95 \% \mathrm{Cl}\end{array}$}} & \multirow{2}{*}{$\begin{array}{l}\text { Risk of bias } \\
\text { A B C D F G }\end{array}$} \\
\hline & Events & Total & Events & Total & & & & & & \\
\hline Gungor et al..$^{18}(2015)$ & 62 & 100 & 71 & 100 & 34.6 & $0.87(0.72,1.06)$ & \multirow{2}{*}{\multicolumn{3}{|c|}{$\frac{1}{1}$}} & \multirow{2}{*}{ 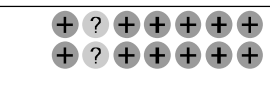 } \\
\hline Kefeli et al. ${ }^{16}(2016)$ & 114 & 130 & 113 & 130 & 65.4 & $1.01(0.92,1.11)$ & & & & \\
\hline Total $(95 \% \mathrm{Cl})$ & & 230 & & 230 & 100.0 & $0.96(0.83,1.12)$ & \multicolumn{3}{|c|}{$\checkmark$} & \\
\hline Total events & 176 & & 184 & & & & & & & + \\
\hline \multicolumn{7}{|c|}{ Heterogeneity: $\operatorname{Tau}^{2}=0.01, \mathrm{chi}^{2}=2.08, \mathrm{df}=1(P=0.15), I^{2}=52 \%$} & 0.01 & 0.1 & 10 & 100 \\
\hline \multicolumn{7}{|c|}{ Test for overall effect: $Z=0.57(P=0.59)$} & \multicolumn{4}{|c|}{ Favours 10-day SQT } \\
\hline
\end{tabular}

\begin{tabular}{|c|c|c|c|c|c|c|c|c|c|c|}
\hline \multirow[b]{2}{*}{ Study or subgroup } & \multicolumn{2}{|c|}{ 10-/14-day PBMT } & \multicolumn{2}{|c|}{ 10-day SQT } & \multirow{2}{*}{$\begin{array}{l}\text { Weight } \\
(\%)\end{array}$} & \multirow{2}{*}{$\begin{array}{c}\text { Risk ratio } \\
\mathrm{M}-\mathrm{H}, \text { random, } 95 \% \mathrm{Cl}\end{array}$} & \multirow{2}{*}{\multicolumn{3}{|c|}{$\begin{array}{c}\text { Risk ratio } \\
\mathrm{M}-\mathrm{H} \text {, random, } 95 \% \mathrm{Cl}\end{array}$}} & \multirow{2}{*}{$\begin{array}{l}\text { Risk of bias } \\
\text { A B C D E F G }\end{array}$} \\
\hline & Events & Total & Events & Total & & & & & & \\
\hline Gungor et al. $^{18}(2015)$ & 62 & 80 & 71 & 88 & 13.1 & $0.96(0.82,1.12)$ & & & & \multirow{2}{*}{ 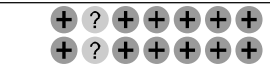 } \\
\hline Kefeli et al. ${ }^{16}(2016)$ & 114 & 121 & 113 & 119 & 86.9 & $0.99(0.93,1.05)$ & & & & \\
\hline Total $(95 \% \mathrm{Cl})$ & & 201 & & 207 & 100.0 & $0.99(0.93,1.05)$ & & 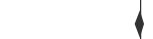 & & \\
\hline Total events & 176 & & 184 & & & & & & & + \\
\hline \multicolumn{7}{|c|}{ Heterogeneity: $\mathrm{Tau}^{2}=0.00, \mathrm{chi}^{2}=0.22, \mathrm{df}=1(P=0.64), I^{2}=0 \%$} & 0.01 & 0.1 & 10 & 100 \\
\hline \multicolumn{5}{|c|}{ Test for overall effect: $Z=0.42(P=0.67)$} & \multicolumn{6}{|c|}{ Favours 10-114-day $\quad$ Favours 10-day SQT } \\
\hline
\end{tabular}

(B)

Fig. 3. Forest plot of Helicobacter pylori eradication of 10- or 14-day bismuth-containing quadruple therapy (PBMT) vs. 10-day sequential therapy (SQT) as the first-line therapy. (A) Intention-to-treat analysis. (B) Per-protocol analysis. Risk of bias: A, random sequence generation (selection bias); B, allocation concealment (selection bias); C, blinding of participants and personnel (performance bias); D, blinding of outcome assessment (detection bias); E, incomplete outcome data (attrition bias); F, selective reporting (reporting bias); G, other bias. M-H, Mantel-Haenszel test.

77.1 94.9\%) in PP analysis. The pooled eradication rates of non-PBMT were $77.7 \%$ (95\% CI, 62.7 88.0\%) and 83.3\% (95\% CI, 68.0 92.1\%) in ITT and PP analyses, respectively. In terms of the treatment duration, the pooled eradication rates of 10-day PBMT were $79.6 \%$ (95\% CI, 47.4 94.4\%) and 88.6\% (95\% CI, 54.7 98.0\%) in ITT and PP analyses, respectively. The pooled eradication rates of 14-day PBMT were 83.9\% (95\% CI, 66.9 93.1\%) in ITT analysis and 88.9\% (95\% CI, 77.2 95.0\%) in PP analysis.

\section{Eradication rates between PBMT and standard triple therapy}

Of three trials, 759 patients were prescribed PBMT for 10 or 14 days, and 753 patients received standard triple therapy for 14 days (Fig. 2). When we compared eradication rates between 10- or 14-day PBMT and 14-day standard triple therapy, the differences were not statistically significant in ITT and PP analyses (ITT analysis: RR, 1.28; 95\% CI, 0.97 1.70; $P=0.08$; PP analysis: RR, $1.37 ; 95 \% \mathrm{CI}$,
0.95 1.99; $P=0.10$ ). However, heterogeneity among the studies was high in the ITT and PP analyses (ITT analysis: $\chi^{2}=10.14, P=0.006, I^{2}=80 \%$; PP analysis: $\chi^{2}=20.95, P<0.001$, $\left.I^{2}=90 \%\right)$.

\section{Eradication rates between PBMT and SQT}

Each of 230 patients were treated with 10- or 14-day PBMT and 10-day SQT (Fig. 3). Eradication rates between 10- or 14-day PBMT and 10-day SQT were not significantly different in ITT and PP analyses (ITT analysis: RR, 0.96; 95\% CI, 0.83 1.12; $P=0.59$; PP analysis: RR, 0.99; 95\% CI, 0.93 1.05; $P=0.67$ ). Heterogeneity between the studies was moderate in ITT analysis $\left(x^{2}=2.08\right.$, $\left.P=0.15, I^{2}=52 \%\right)$, and low in PP analysis $\left(\chi^{2}=0.22, P=0.64\right.$, $I^{2}=0 \%$.

\section{Eradication rates between PBMT and CT}

Each of 770 patients received PBMT for 10 or 14 days and CT for 10 days (Fig. 4). There were no significant dif- 
ferences between 10- or 14-day PBMT and 10-day CT groups in either analyses (ITT analysis: RR, 1.01; 95\% CI, 0.93 1.10; $P=0.83$; PP analysis: RR, $1.01 ; 95 \% \mathrm{CI}$,
0.95 1.07; $P=0.83$ ). Moderate heterogeneity in the studies was revealed in both the ITT and PP analyses (ITT analysis: $\chi^{2}=4.57, P=0.10, I^{2}=56 \%$; PP analysis: $\chi^{2}=5.51$,

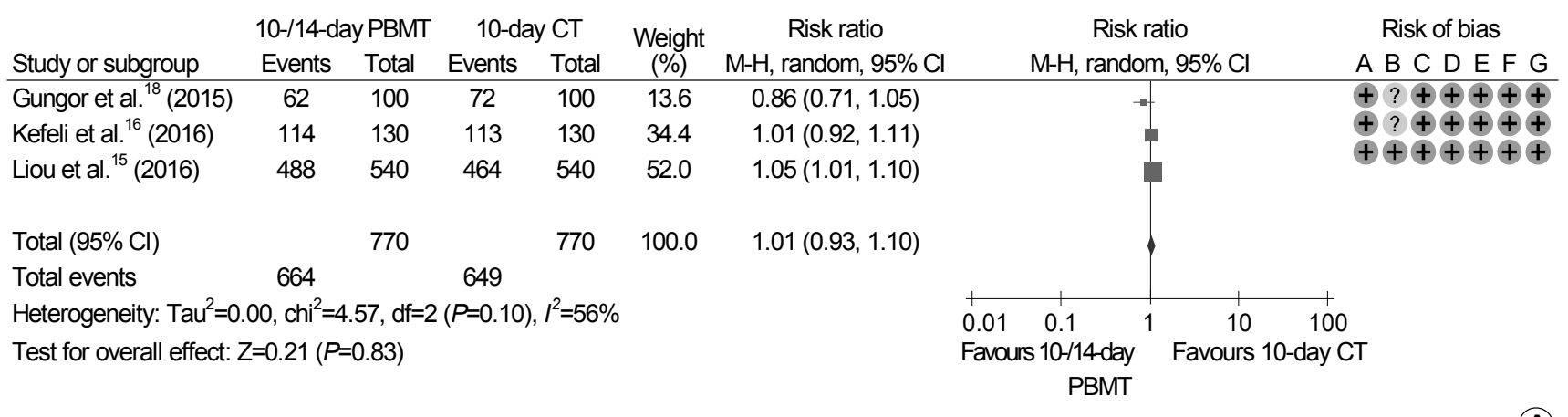

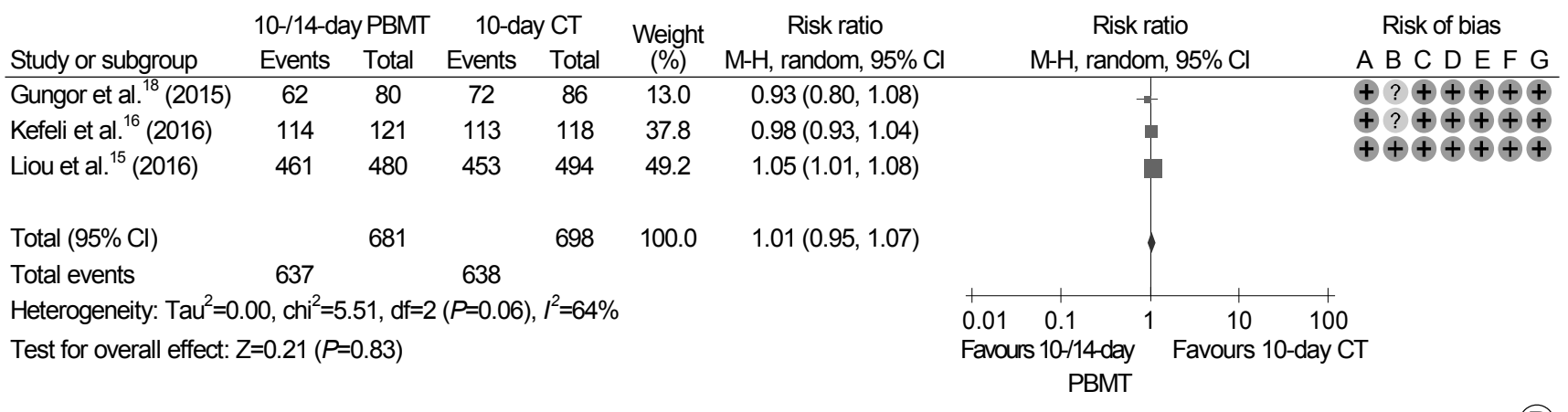

Fig. 4. Forest plot of Helicobacter pylori eradication with 10- or 14-day bismuth-containing quadruple therapy (PBMT) vs. 10-day concomitant therapy (CT) as the first-line therapy. (A) Intention-to-treat analysis. (B) Per-protocol analysis. Risk of bias: A, random sequence generation (selection bias); B, allocation concealment (selection bias); C, blinding of participants and personnel (performance bias); D, blinding of outcome assessment (detection bias); E, incomplete outcome data (attrition bias); F, selective reporting (reporting bias); G, other bias. M-H, Mantel-Haenszel test.

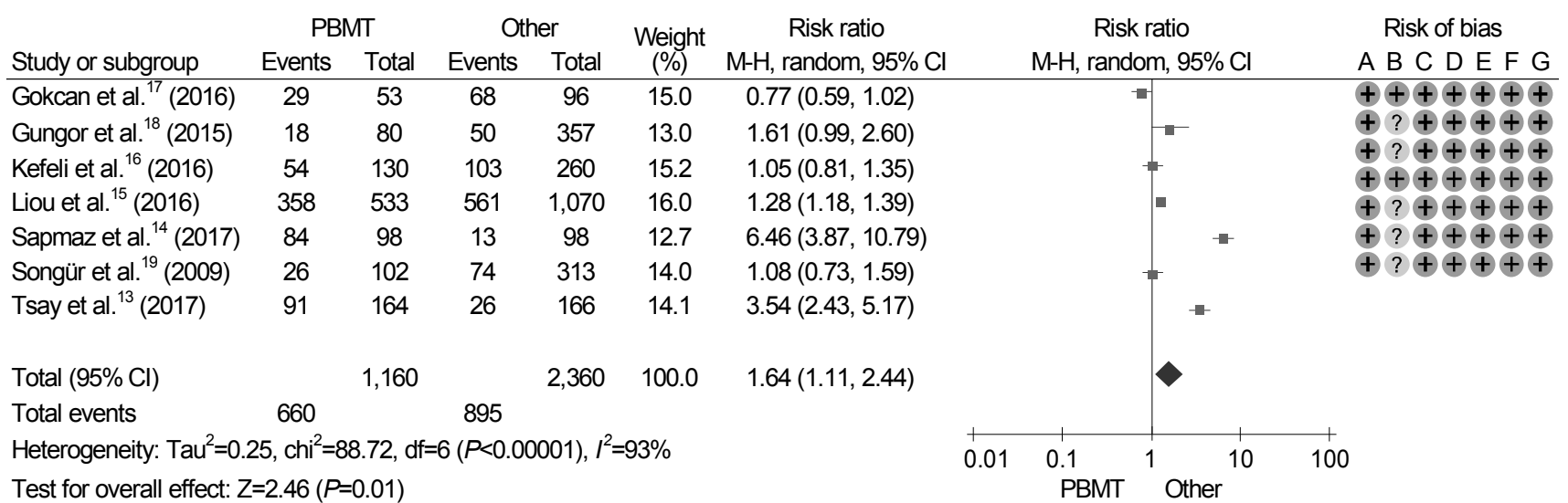

Fig. 5. Forest plot of Helicobacter pylori eradication with bismuth-containing quadruple therapy (PBMT) vs. non-bismuth-containing quadruple therapy as the first-line therapy. Risk of bias: A, random sequence generation (selection bias); B, allocation concealment (selection bias); C, blinding of participants and personnel (performance bias); D, blinding of outcome assessment (detection bias); E, incomplete outcome data (attrition bias); F, selective reporting (reporting bias); G, other bias. M-H, Mantel-Haenszel test. 
$P=0.06, I^{2}=64 \%$.

\section{Adverse events}

All seven trials identified adverse events with treatment. With regard to the duration of the treatment, the pooled adverse event rate of 10-day PBMT were 44.6\% (95\% CI, 22.5 69.1\%) and those of 14-day PBMT were 55.9\% (95\% CI, 27.6 80.8\%). The meta-analysis of these trials revealed that the frequency of adverse events was significantly higher in patients with PBMT than in those with non-PBMT, and the pooled RR for adverse events was 1.64 (95\% CI, 1.11 2.44; P=0.01) (Fig. 5). However, significant heterogeneity in adverse event was observed in the trials $\left(\chi^{2}=88.7, P<0.001, I^{2}=93 \%\right)$.

\section{DISCUSSION}

Eradication rate of $H$. pylori is closely related to antibiotics resistance. Among them, the resistance of clarithromycin reduces the success rate of $H$. pylori eradication. A recent meta-analysis reported that the pooled RR of the eradication rate was $0.682(95 \% \mathrm{CI}, 0.636 \sim 0.731)$ in patients with $H$. pylori strains sensitive versus resistant to clarithromycin, whereas the pooled $\mathrm{RR}$ of the eradication rate was 0.843 $(95 \%$ CI, 0.810 0.877) and 0.794 (95\% CI, 0.669 0.941) in patients with $H$. pylori strains sensitive versus resistant to metronidazole and levofloxacin, respectively. ${ }^{20}$ Compared to other antibiotics, clarithromycin had the most pervasive influence in eradication rates.

High clarithromycin resistance areas are commonly defined as the clarithromycin resistance rate of $H$. pylori, which is at least $15 \% .^{7}$ Clarithromycin resistance has increased during the last decade in Korea, ${ }^{21}$ and recent Korean studies still presented that clarithromycin resistance rates were $17.8 \sim 31.0 \%{ }^{22,23}$ At least for first-line $H$. pylori eradication therapy, successful eradication rates should be more than $80 \%$ in ITT analysis and more than 90\% in PP analysis. ${ }^{24}$ Established guidelines recommended that bismuth-containing quadruple or non-bismuth-containing quadruple therapies are appropriate as the first-line $H$. pylori eradication therapy in areas of high clarithromycin resistance. ${ }^{7,8}$ This meta-analysis revealed that the pooled eradication rate for PBMT as a first-line eradication therapy was $82.1 \%$ and $88.8 \%$ in ITT and PP analyses. Although the eradication rate in PP analysis did not reach $90 \%$, the effect of PBMT was assumed to be sufficient because the eradication rate was almost 90\%.

Nonetheless, when we compared the eradication rates between PBMT and other widely used first-line eradication regimens, there were no statistically significant differences of eradication rates in 10- or 14-day PBMT with 10-day SQT (RR, 0.99; 95\% CI, 0.93 1.05), 10-day CT (RR, 1.01; 95\% CI, 0.95 1.07), or even 14-day standard triple therapy (RR, 1.37; 95\% CI, 0.95 1.99). Our results indicate that 14-day standard triple therapy, 10-day SQT, and 10-day CT are not inferior to 10- or 14-day PBMT.

Duration of treatment can affect the H. pylori eradication rate, and regimens of longer duration had higher eradication rates than did regimens of shorter duration. ${ }^{10}$ In terms of standard triple therapy, 14-day standard triple therapy (OR, 1.72; 95\% CI, 1.37 2.17) and 10-day standard triple therapy (OR, 1.32; 95\% CI, 1.04 1.69) showed a higher eradication rate than did 7-day standard triple therapy. ${ }^{10}$ Several meta-analyses have demonstrated similar results for standard triple therapy. ${ }^{7,925}$ Although the eradication rates of standard triple therapy tend to decrease, 14-day standard triple therapy could be an acceptable regimen, since it has a higher therapeutic effect than does standard triple therapy with a shorter duration.

The results obtained in our study comparing PBMT and SQT are consistent with previous meta-analyses. According to the systematic review and meta-analysis study which analyzed 46 RCTs with 13,532 patients, the overall eradication rate of SQT was $84.3 \%(95 \% \mathrm{CI}, 82.1 \sim 86.4 \%)^{26}$ SQT was superior to 7-day standard triple therapy (RR, $1.21 ; 95 \% \mathrm{CI}$, 1.17 1.25) and 10-day standard triple therapy (RR, 1.11; 95\% CI, 1.04 1.19). ${ }^{26}$ However, SQT was not superior to 14-day standard triple therapy (RR, 1.00; 95\% CI, 0.94 1.06) and 10- or 14-day of bismuth-containing quadruple therapy (RR, 1.01; $95 \%$ CI, 0.95 1.06). ${ }^{26}$

Two prospective studies comparing PBMT and CT as first-line treatment were recently published, and CT showed high eradication rates in both studies. A study from Spain reported that the eradication rate was 98.0\% 
(95\% CI, 94 100\%) in a 14-day CT group and 94.4\% (95\% CI, 88.1 100\%) in a 10-day PBMT group at ITT analysis $(P=0.346){ }^{27}$ There was no significant difference in adverse events rates between CT (56.0\%) and PBMT (46.3\%) $(P=0.323) .^{27}$ The other was a Korean study that conducted a prospective open-label randomized trial comparing 14-day modified PBMT and 14-day $\mathrm{CT}^{28}$ The ITT eradication rate was $88.2 \%$ in a modified PBMT group and $79.4 \%$ in a CT group $(P=0.162){ }^{28} \mathrm{PP}$ eradication rates were $98.4 \%$ and $93.1 \%$ in the modified PBMT and CT groups, respectively $(P=0.199) .{ }^{28}$ Adverse events rates were higher in CT (51.5\%) than in modified PBMT (33.8\%) patients $(P=0.037){ }^{28}$ The adverse event rates of both studies were higher in the CT group than in the PBMT group; however, it should be considered that the duration of CT is longer than that of PBMT in the Spanish study, and the Korean study used modified PBMT that was prescribed PBMT twice a day. A meta-analysis of 19 clinical trials with 2,070 patients reported that a mean ITT cure rate was $88 \%$ (95\% CI, 85 91\%) for $\mathrm{CT}^{29}$

It is already known that adverse events of PBMT are common. The adverse events rates of PBMT typically arise in $50 \%$ or more. ${ }^{30}$ The rates of adverse events were $67 \%$ (358/533) in 10-day PBMT, 58\% (309/535) in 10-day CT, and $47 \%(252 / 535)$ in 14-day standard triple therapy groups in a multi-center, randomized trial from Taiwan. ${ }^{15}$ Although we observed considerable heterogeneity among trials, the adverse events of PBMT were significantly higher than in other eradication therapies (RR, 1.64; 95\% CI, 1.11 2.44) in the current meta-analysis. A recent network meta-analysis including 35 studies with 10,860 patients for demonstrating adverse events reported that PBMT showed more adverse events than did SQT and CT. ${ }^{31}$ Compared to 14-day PBMT, 14-day SQT (OR, 0.42; 95\% CI, 0.22 0.80), 10-day SQT (OR, 0.49; 95\% CI, 0.31 0.78), and 10- or 14-day CT (OR, 0.57; 95\% CI, 0.37 0.89) therapies were significantly lower in the adverse events rates. ${ }^{31}$ There was no significant difference in rates of adverse events between PBMT and standard triple therapy groups in other meta-analyses; however, the overall incidence of adverse events was 46\% in PBMT and 46.3\% in the standard triple therapy groups. ${ }^{32}$ Therefore, the adverse events of PBMT are an issue to consider in clinical practice when using PBMT regimens.

Another problem to take account of in prescribing PBMT is rescue therapy related. It is difficult to decide on a rescue therapy after failure of PBMT. The Maastricht V/Florence consensus report recommended a quinolone-containing triple or quadruple therapy in patients who failed with PBMT. $^{7}$ In high quinolone-resistance areas, it is recommended to use the combination of bismuth with other antibiotics. ${ }^{7}$ The revised Korean guideline recommended a rescue regimen containing two or more antibiotics that had not been included in PBMT in patients with eradication failure for $\mathrm{PBMT}^{3}$ Given the quinolone resistance to $H$. pylori, the resistance rates of both levofloxacin and moxifloxacin revealed a tendency to increase time-dependently $(4.7 \sim 28.1 \%, P=0.002)$ in Korea. $^{21}$ A recent Korean study showed that the resistance rates of both levofloxacin and ciprofloxacin were 37.0\%. ${ }^{33}$ Another study from Korea compared 7-day PBMT with 14-day moxifloxacin triple therapy as a second-line $H$. pylori eradication therapy. ${ }^{34}$ Eradication rates were 93.6\% (95\% CI, 91.0 95.9\%) and 73.8\% (95\% CI, 63.1 84.6\%) in 7-day PBMT and 14-day moxifloxacin triple therapy groups, respectively $(P<0.001){ }^{34}$ Despite the shorter duration of PBMT, it exhibited a higher eradication rate than did the longer duration of moxifloxacin triple therapy. H. pylori culture and antimicrobial susceptibility testing are preferred methods to be used after eradication failure of PBMT regimens. ${ }^{7}$ Actually, few laboratories have been installed with these systems in Korea. Thus, though PBMT is a valuable treatment for $H$. pylori infection, PBMT as a first-line therapy could be recommended for patients with an allergy to penicillin, with a clarithromycin-resistant $H$. pylori infection, or with a history of previous macrolide use.

This study has several limitations. First, only a few trials were included in our analysis, and heterogeneities among studies were generally moderate or high. Therefore, this should be considered when interpreting our results. Second, studies from Korea could not be analyzed because there are no randomized controlled Korean studies on this subject. However, several Turkish and Taiwanese studies were included in this analysis. Turkey belonged to areas of high H. pylori prevalence $(77.2 \%)^{1}$ and high clarithromycin resist- 
ance (28\%). ${ }^{35}$ The prevalence of $H$. pylori infection in Taiwan was $53.9 \%$ and clarithromycin resistance was $26 \% .35$ Therefore, these results could reflect those in Korea.

In conclusion, PBMT can be a first-line treatment for H. pylori eradication when other first-line options are unavailable, because of its high adverse events rate and its potential for use as rescue therapy. Further well-designed studies are needed to prove the efficacy of PBMT for first-line $H$. pylori eradication in Korea.

\section{CONFLICTS OF INTEREST}

Sung Eun Kim is an editorial board member of the Journal but did not involve in the peer reviewer selection, evaluation, or decision process of this article. No other potential conflicts of interest relevant to this article were reported.

\section{ORCID}

\begin{tabular}{|c|c|}
\hline n & 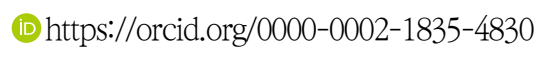 \\
\hline ng Jung & (D) https://orcid.org/0000-0002-6653-5214 \\
\hline Joo Kang & (D) https://orcid.org/0000-0002-7401-8356 \\
\hline Chan Lee & 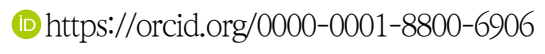 \\
\hline ang & \\
\hline Young Park & (D) https://orcid.org/0000-0002-0962 \\
\hline leol Min Shin & (1) https://orcid.org/0000-0003- \\
\hline Chul Lim & (1D) https://orcid.c \\
\hline -Hyun Kim & g 70000 \\
\hline Youn Nam & (D) https://orcid.org/0000-0002 \\
\hline oon Geon Shin & - \\
\hline Myung Park & (D) https://orci \\
\hline Ju Choi & $\mathrm{g} / 0000-000$ \\
\hline & 15 600 \\
\hline & \\
\hline
\end{tabular}

\section{REFERENCES}

1. Hooi JKY, Lai WY, Ng WK, et al. Global prevalence of Helicobacter pylori infection: systematic review and metaanalysis. Gastroenterology 2017;153:420-429.

2. Moss SF, Malfertheiner P. Helicobacter and gastric malignancies. Helicobacter 2007;12 Suppl 1:23-30.

3. Kim SG, Jung HK, Lee HL, et al. Guidelines for the diagnosis and treatment of Helicobacter pylori infection in Korea, 2013 revised edition. J Gastroenterol Hepatol 2014;29:1371-1386.

4. Sasaki M, Ogasawara N, Utsumi K, et al. Changes in 12-year first-line eradication rate of Helicobacter pylori based on triple therapy with proton pump inhibitor, amoxicillin and clarithromycin. J Clin Biochem Nutr 2010;47:53-58.

5. Gong EJ, Yun SC, Jung HY, et al. Meta-analysis of first-line triple therapy for Helicobacter pylorieradication in Korea: is it time to change? J Korean Med Sci 2014;29:704-713.

6. Kim SE, Park MI, Park SJ, et al. Trends in Helicobacter pylori eradication rates by first-line triple therapy and related factors in eradication therapy. Korean J Intern Med 2015;30:801-807.

7. Malfertheiner P, Megraud F, O'Morain CA, et al. Management of Helicobacter pylori infection-the Maastricht V/Florence consensus report. Gut 2017;66:6-30.

8. Chey WD, Leontiadis GI, Howden CW, Moss SF. ACG clinical guideline: treatment of Helicobacter pylori infection. Am J Gastroenterol 2017;112:212-239.

9. Li BZ, Threapleton DE, Wang JY, et al. Comparative effectiveness and tolerance of treatments for Helicobacter pylori: systematic review and network meta-analysis. BMJ 2015;351:h4052.

10. Yeo YH, Shiu SI, Ho HJ, et al. First-line Helicobacter pylorieradication therapies in countries with high and low clarithromycin resistance: a systematic review and network meta-analysis. Gut 2018;67:20-27.

11. Liberati A, Altman DG, Tetzlaff J, et al. The PRISMA statement for reporting systematic reviews and meta-analyses of studies that evaluate health care interventions: explanation and elaboration. J Clin Epidemiol 2009;62:e1-e34.

12. Higgins JPT, Altman DG, Sterne JAC. Cochrnae handbook for systematic reviews of interventions, version 5.1.0. Chapter 8: assessing risk of bias in included studies [Internet]. London: Cochrane Training; 2006 [updated 2011 Mar; cited 2019 May 10]. Available from: http://handbook.cochrane.org/chapter_8/8_ assessing_risk_of_bias_in_included_studies.html.

13. Tsay FW, Wu DC, Yu HC, et al. A randomized controlled trial shows that both 14-day hybrid and bismuth quadruple therapies cure most patients with Helicobacter pylori infection in populations with moderate antibiotic resistance. Antimicrob Agents Chemother 2017;61:e00140-17.

14. Sapmaz F, Kalkan IH, Atasoy P, Basyigit S, Guliter S. A non-inferiority study: modified dual therapy consisting higher doses of rabeprazole is as successful as standard quadruple therapy in eradication of Helicobacter pylori. Am J Ther 2017;24:e393e398.

15. Liou JM, Fang YJ, Chen CC, et al. Concomitant, bismuth quadruple, and 14-day triple therapy in the first-line treatment of Helicobacter pylori: a multicentre, open-label, randomised trial. Lancet 2016;388:2355-2365.

16. Kefeli A, Basyigit S, Yeniova AO, Kefeli TT, Aslan M, Tanas O. Comparison of three different regimens against Helicobacter pylori as a first-line treatment: a randomized clinical trial. Bosn J Basic Med Sci 2016;16:52-57.

17. Gokcan H, Oztas E, Onal IK. Different bismuth-based therapies 
for eradicating Helicobacter pylori: randomized clinical trial of efficacy and safety. Clin Res Hepatol Gastroenterol 2016;40: 124-131.

18. Gungor G, Baglicakoglu M, Kayacetin E, et al. Current status of five different regimens for empiric first-line Helicobacter pylori eradication in Turkey. Digestion 2015;92:55-59.

19. Songür Y, Senol A, Balkarli A, Baştürk A, Cerçi S. Triple or quadruple tetracycline-based therapies versus standard triple treatment for Helicobacter pylori treatment. Am J Med Sci 2009;338: 50-53.

20. Zou Y, Qian X, Liu X, et al. The effect of antibiotic resistance on Helicobacter pylori eradication efficacy: a systematic review and meta-analysis. Helicobacter 2020;25:e12714.

21. Lee JW, Kim N, Kim JM, et al. Prevalence of primary and secondary antimicrobial resistance of Helicobacter pylori in Korea from 2003 through 2012. Helicobacter 2013;18:206-214.

22. Lee JH, Ahn JY, Choi KD, et al. Nationwide antibiotic resistance mapping of Helicobacter pylori in Korea: a prospective multicenter study. Helicobacter 2019;24:e12592.

23. Lee JW, Kim N, Nam RH, et al. Favorable outcomes of culture-based Helicobacter pylori eradication therapy in a region with high antimicrobial resistance. Helicobacter 2019;24:e12561.

24. Graham DY, Fischbach L. Helicobacter pylori treatment in the era of increasing antibiotic resistance. Gut 2010;59:1143-1153.

25. Calvet X, García N, López T, Gisbert JP, Gené E, Roque M. A meta-analysis of short versus long therapy with a proton pump inhibitor, clarithromycin and either metronidazole or amoxycillin for treating Helicobacter pylori infection. Aliment Pharmacol Ther 2000;14:603-609.

26. Gatta L, Vakil N, Vaira D, Scarpignato C. Global eradication rates for Helicobacter pylori infection: systematic review and meta-analysis of sequential therapy. BMJ 2013;347:f4587.

27. Macías-García F, Bastón-Rey I, de la Iglesia-García D, Calviño-Suárez C, Nieto-García L, Domínguez-Muñoz JE. Bismuth-containing quadruple therapy versus concomitant quadruple therapy as first-line treatment for Helicobacter pylori infection in an area of high resistance to clarithromycin: a prospective, cross-sectional, comparative, open trial. Helicobacter 2019;24:e12546.

28. Kim SJ, Chung JW, Woo HS, et al. Two-week bismuth-containing quadruple therapy and concomitant therapy are effective first-line treatments for Helicobacter pylori eradication: a prospective open-label randomized trial. World J Gastroenterol 2019;25:6790-6798.

29. Gisbert JP, Calvet X. Update on non-bismuth quadruple (concomitant) therapy for eradication of Helicobacter pylori. Clin Exp Gastroenterol 2012;5:23-34.

30. Graham DY, Lee SY. How to effectively use bismuth quadruple therapy: the good, the bad, and the ugly. Gastroenterol Clin North Am 2015;44:537-563.

31. Xu H, Wang W, Ma X, et al. Comparative efficacy and safety of high-dose dual therapy, bismuth-based quadruple therapy and non-bismuth quadruple therapies for Helicobacter pylori infection: a network meta-analysis. Eur J Gastroenterol Hepatol 2020. doi: 10.1097/MEG.0000000000001835. [Epub ahead of print]

32. Venerito M, Krieger T, Ecker T, Leandro G, Malfertheiner P. Meta-analysis of bismuth quadruple therapy versus clarithromycin triple therapy for empiric primary treatment of Helicobacter pylori infection. Digestion 2013;88:33-45.

33. Lee JH, Ahn JY, Choi KD, et al. Nationwide antibiotic resistance mapping of Helicobacter pylori in Korea: a prospective multicenter study. Helicobacter 2019;24:e12592.

34. Kim SE, Roh JH, Park MI, et al. Effect of 7-day bismuth quadruple therapy versus 14-day moxifloxacin triple therapy for second-line Helicobacter pylori eradication therapy. Korean J Gastroenterol 2019;73:26-34.

35. Savoldi A, Carrara E, Graham DY, Conti M, Tacconelli E. Prevalence of antibiotic resistance in Helicobacter pylori: a systematic review and meta-analysis in World Health Organization regions. Gastroenterology 2018;155:1372-1382.e17. 